\title{
Analysis and discussion of a sample of 25 gigahertz-peaked spectra pulsars
}

\author{
Jarosław Kijak, Wojciech Lewandowski and Karolina Rożko \\ Janusz Gil Institute of Astronomy, University of Zielona Góra, \\ ul. Szafrana 2, 65-516 Zielona Góra, Poland \\ email: J.Kijak@ia.uz.zgora.pl
}

\begin{abstract}
We identified gigahertz-peaked spectra behavior from our radio interferometric observations at low frequencies using the Giant Metrewave Radio Telescope. We modeled the turnover spectra based on thermal free-free absorption in the interstellar medium. The free-free absorption is believed to be responsible for the inverted spectrum. Using the model, we were able to put some observational constrains on the physical parameters of the absorbing matter, which allows us to distinguish between the possible sources of absorption.
\end{abstract}

Keywords. pulsars, radio spectra

In recent years a new class of pulsars has been identified with a distinct spectral nature showing a turnover around $1 \mathrm{GHz}$. These sources, named the gigahertz-peaked spectra (GPS) pulsars, exhibit the typical power law spectrum at higher frequencies, but at low frequencies their observed flux decreases with frequency and the corresponding spectral index becomes positive. The GPS phenomenon in pulsars was first identified by Kijak, Gupta \& Krzeszowski (2007) and have subsequently been established as a separate group within the pulsar population by Kijak et al. (2011a, b, 2013), Dembska et al. (2014). Using the newly acquired interferometric data and the previously published flux measurements we constructed the spectra for all pulsars that either exhibit the GPS behavior or had been suspected to do so before our recent observations. So far 25 GPS pulsars were identified, a number that is steadily growing over the past few years Kijak et al. $(2017 \mathrm{a}, \mathrm{b})$.

It is very likely, that the GPS phenomenon in pulsars is a result of thermal absorption of the pulsar flux in peculiar neutron star environments or in the dense interstellar medium along the line of sight. The idea of the thermal free-free absorption as the source of the low frequency spectral turnovers in pulsars was first proposed by Sieber (1973) and later was proposed to explain the GPS spectra of pulsars and magnetars by Kijak et al. (2011a, 2013). A detailed study was conducted by Lewandowski et al. (2015) and Basu et al. (2016), Rajwade, Lorimer \& Anderson (2016), where the low frequency turnover in the spectral shape was successfully modeled using thermal absorption. A comprehensive analysis of the currently known GPS pulsars using the model is shown in Kijak et al. (2017a, b). The appearance of the GPS spectrum is related to the peculiar environments (such as PWNs, HII regions, etc.) around these objects (see also Rożko et al. in the same proceedings). Here, we present a sample of 25 gigahertz-peaked spectra pulsars in Table 1 and examples of new GPS pulsars in Figure 1.

\section{Acknowledgement}

We thank the staff of the GMRT who have made these observations possible. The GMRT is run by the National Centre for Radio Astrophysics of the Tata Institute 

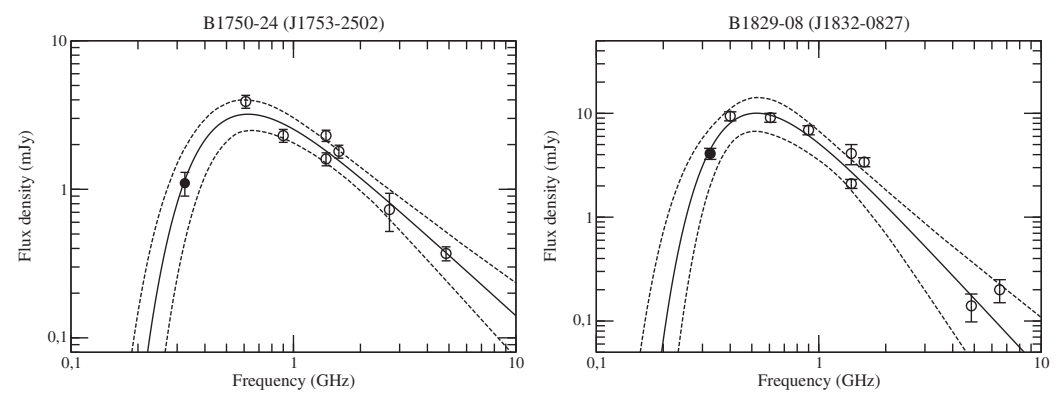

Figure 1. Examples of new GPS pulsars (Kijak et al. 2017b). The empty symbols show flux from the literature. Our measurements were denoted by filled circles. The solid line represents the thermal absorption model fit for the observed data with $1 \sigma$ envelope (dashed lines).

Table 1. The remarks column indicates definite or possible associations of the neutron star with a supernova remnant nebula (SNR), a pulsar wind nebula (PWN), an H II region, or an unidentified X-ray source from the HESS catalog.

\begin{tabular}{|c|c|c|c|}
\hline PSR & $\begin{array}{c}\mathrm{DM} \\
\left(\mathrm{pc} \mathrm{cm}^{-3}\right)\end{array}$ & $\begin{array}{l}\text { Age } \\
\text { (kyr) }\end{array}$ & ASSOCIATION \\
\hline B1054-62 & 320 & 1870 & H II (BBW 328) \\
\hline B1259-63 & 146 & 332 & Be star \\
\hline $\mathrm{J} 1550-5418$ & 830 & 1.41 & Radiomagnetar, SNR \\
\hline J1622-4950 & 820 & 4.03 & Radiomagnetar, SNR, PWN?, H II \\
\hline B1641-45 & 479 & 359 & HESS, (near Westerlund 1 globular cluster) \\
\hline J1723-3659 & 254 & 401 & \\
\hline J1739-3023 & 170.0 & 0.16 & PWN \\
\hline $\mathrm{J} 1745-2900$ & 1778 & 3.40 & Radiomagnetar, SGR A* \\
\hline B1750-24 & 672.0 & 0.59 & \\
\hline B1754-24 & 179 & 0.29 & $3 \mathrm{EG}$ \\
\hline B1800-21 & 234 & 15.8 & SNR, HII (W30) \\
\hline J1809-1917 & 197 & 51.3 & X:bow shock PWN SNR?, H II?, HESS \\
\hline B1820-14 & 651 & 3.75 & \\
\hline B $1822-14$ & 357 & 195 & SNR, H II \\
\hline B1823-13 & 231 & 21.4 & $\mathrm{X}: \mathrm{PWN}$ \\
\hline B1829-08 & 300 & 0.16 & XRS:PWN \\
\hline B1830-08 & 411 & 0.15 & XRS:PWN \\
\hline $\mathrm{J} 1835-1020$ & 114 & 810 & \\
\hline B1838-04 & 325 & 461 & $3 \mathrm{EG}$ \\
\hline $\mathrm{J} 1841-0345$ & 194 & 55.9 & H II? \\
\hline $\mathrm{J} 1852-0635$ & 171 & 567 & \\
\hline $\mathrm{J} 1901+0510$ & 429 & 313 & \\
\hline $\mathrm{J} 1905+0616$ & 256 & 0.12 & SNR? \\
\hline $\mathrm{J} 1907+0918$ & 358 & 38 & SNR? \\
\hline $\mathrm{J} 2007+2722$ & 127 & 404000 & \\
\hline
\end{tabular}

of Fundamental Research. This research was partially supported by the grant DEC2013/09/B/ST9/02177 of the Polish National Science Centre.

\section{References}

Basu, R., Rożko, K., Lewandowski, W., Kijak, J., \& Dembska, M. 2016, MNRAS, 458, 2509

Dembska M., Kijak J., Lewandowski W. et al. 2014, MNRAS, 445, 3105

Kijak, J., Gupta, Y., \& Krzeszowski, K. 2007, A $\mathscr{G} A$, 462, 699-702

Kijak, J., Dembska, M., Lewandowski, W. et al. 2011a, MNRAS (Letters), 418, L114-L118

Kijak, J., Lewandowski, W., Maron, O., Gupta, Y., \& Jessner, A. 2011b, A 6 A, 531, A16

Kijak, J., Tarczewski, L., Lewandowski, W., \& Melikidze, G. 2013, ApJ, 772, 29

Kijak, J., Basu, R., Lewandowski, W., Rożko, K., \& Dembska M. 2017a, ApJ, 840, 108

Kijak, J., Basu, R., Lewandowski, W., \& Rożko, K. 2017b, in preparation

Lewandowski, W., Rożko, K., Kijak, J., \& Melikidze, G. I. 2015, ApJ, 808, 18

Rajwade, K., Lorimer, D. R., \& Anderson, L. D. 2016, MNRAS, 455, 493

Sieber, W. 1973, A\&\&A, 28, 237 